EPiC Series in Engineering
Volume 3, 2018, Pages 643-650
HIC 2018. 13th International
Conference on Hydroinformatics

\title{
Estimation of Longitudinal and Transverse Dispersion Coefficients in Saturated Porous Media Involving Physical Model
}

\author{
Kumars Ebrahimi ${ }^{1}$, Mohammad Reza Nazem ${ }^{1}$, Zahra Mardani ${ }^{1 *}$, Shahab \\ Araghinejad $^{1}$ and Abdolmajid Liaghat ${ }^{1}$ \\ ${ }^{1}$ University of Tehran, Tehran, IRAN \\ Ebrahimikeut.ac.ir, MR.Nazem1991@ut.ac.ir, \\ Zahra.Mardani95@ut.ac.ir, Araghinejad@ut.ac.ir, \\ ALiaghateut.ac.ir
}

\begin{abstract}
The world's urban population growth, indiscriminate use of fertilizers and chemical poisons are actually threatening groundwater resources. The main target of this paper has been tracking of distribution of a conservative tracer in a porous media and in a laboratory-scale model, to estimate the longitudinal and transverse dispersion coefficients. In this study non-cohesive sands were used to create the porous media body in the laboratory model with a grain diameter of 1-2.5 mm. Salt $(\mathrm{NaCl})$ with concentrations of 5,7.5 and $10 \mathrm{~g} / 1$ were used as a tracer. The results of $5 \mathrm{~g} / \mathrm{l}$ concentration tests are reported in this paper. An EC-meter apparatus was used to measure the EC values of the tracer to monitor its plume migration. Then the collected and recorded EC data were used to calculate the tracer concentration data in different points over time, for each test. The calculated concentration data were compared with values which were obtained from the analytical solution using Fickian second law. According to the results, for instance, in the case of $5 \mathrm{~g} / \mathrm{l}$ of the tracer, the obtained values of the longitudinal and transverse coefficients are equal to $3.36 \mathrm{e}-6$ and $6.58 \mathrm{e}-7 \mathrm{~m} 2 / \mathrm{s}$, respectively.
\end{abstract}

\footnotetext{
${ }^{*}$ Corresponding author of the paper
} 
Estimation of Longitudinal and Transverse Dispersion Coefficients in Saturated ... K. Ebrahimi et al.

\section{Introduction}

Groundwater is the main source of drinking, agricultural and industrial water in many arid and semiarid areas; therefore, its quality has a significant impact on human health and the environment. Groundwater quality has been generally threatened with industrial activities, pests and herbicides, underground reservoirs leaks, oil and gas extraction, and landfill dumping.

Therefore, an important issue facing engineers and experts today is the conservation of groundwater resources from the introduction of pollutants and the reduction of specific pollutants in these valuable resources. In order to control the entry of pollutants into aquifers it is necessary to know the sources of pollution and their transmission and distribution processes in porous media. Pollutants are gradually transferred to the groundwater systems and they are part of the flow. The most important parameters affecting the transfer of pollutants are: density, solubility and viscosity of pollutants.

Several models have been used to study groundwater flow systems. The groundwater collections can be divided into three general groups including physical model, analog model and mathematical models.

Nowadays, using laboratory models to capture the realities and understand the phenomena better and to make the right decision about every phenomenon is very common. On the other hand, mathematical groundwater simulation models are used widely by many researchers in many countries and, by the mean of verification; the predicted data are basically compared to the laboratory data. The laboratory model is a simple explanation of identity or a complex process. The use of the model provides many background questions for quick, precise, and economical responses.

Gaggins et al. (2004) determined the longitudinal and transverse dispersion coefficients of groundwater contamination. The results of their research indicated that for a number less than two, the length of the dispersion decreases with increasing velocity, and for the number of cases greater than two, the longitudinal distribution remains constant. Also, by increasing the number of particles, the amount of transverse dispersion decreases almost uniformly.

McNeil et al. (2006) used an image analysis technique to diffuse pollutants in a heterogeneous porous medium. In their study, in order to reassure the results of the image analysis method, the data conforms to the data of a laboratory model and were calibrated. One of the limitations of the image analysis method is that for each porous medium, a separate calibration curve should be considered, based on the particle size and distribution of different states.

Chitarella et al. (2015), after constructing a physical model and photographing the distribution of pollutants, obtained a relation between the glow emitted from the detector (fluoresin) and its concentration at each point. To verify the validity of the obtained relationship, calibrate the results using a numerical model. The final result of their research is the presentation of a new relationship to determine the longitudinal playback coefficient, which is very consistent with the experimental data of past research.

Fadai et al. (2016) used the least squares criterion and photographic processing technique in a laboratory study and estimated the longitudinal and transverse scatter coefficients for different speeds and grading. The values which they presented are equal to $0.001-0.003 \mathrm{~m} 2 / \mathrm{s}$.

According to the literature, the dispersion coefficients depend essentially on the speed, grain size, pollutant concentration, pollutant type, and so it is not possible to provide a coefficient for use in all states. Even in similar studies, the values of the coefficients were somewhat different. In general, it is recommended that, it be consider a length of 500 meters for a longitudinal dispersion and a width of 600 meters in width for scattering, in the field studies. 
Estimation of Longitudinal and Transverse Dispersion Coefficients in Saturated ... K. Ebrahimi et al.

The main purpose of this study was to trace the contamination migration in the saturated porous media state and in a laboratory scale in order to estimate the relevant longitudinal and transverse dispersion coefficients.

\section{Materials and Methods}

In the present study, a homogeneous and saturated porous media based on hypothetical physical models was designed and developed in the central laboratory at the Irrigation and Reclamation Engineering Department of University of Tehran. Figure 1 illustrates a general view of the laboratory model.

As shown in Figure 1, the constructed laboratory glass model had a length of $1.8 \mathrm{~m}$, a width of $0.7 \mathrm{~m}$ and a height of $0.4 \mathrm{~m}$, and was sited in a level and stable situation. Dimensions of the model were selected based on the results of the initial experiments and our previous experiences.

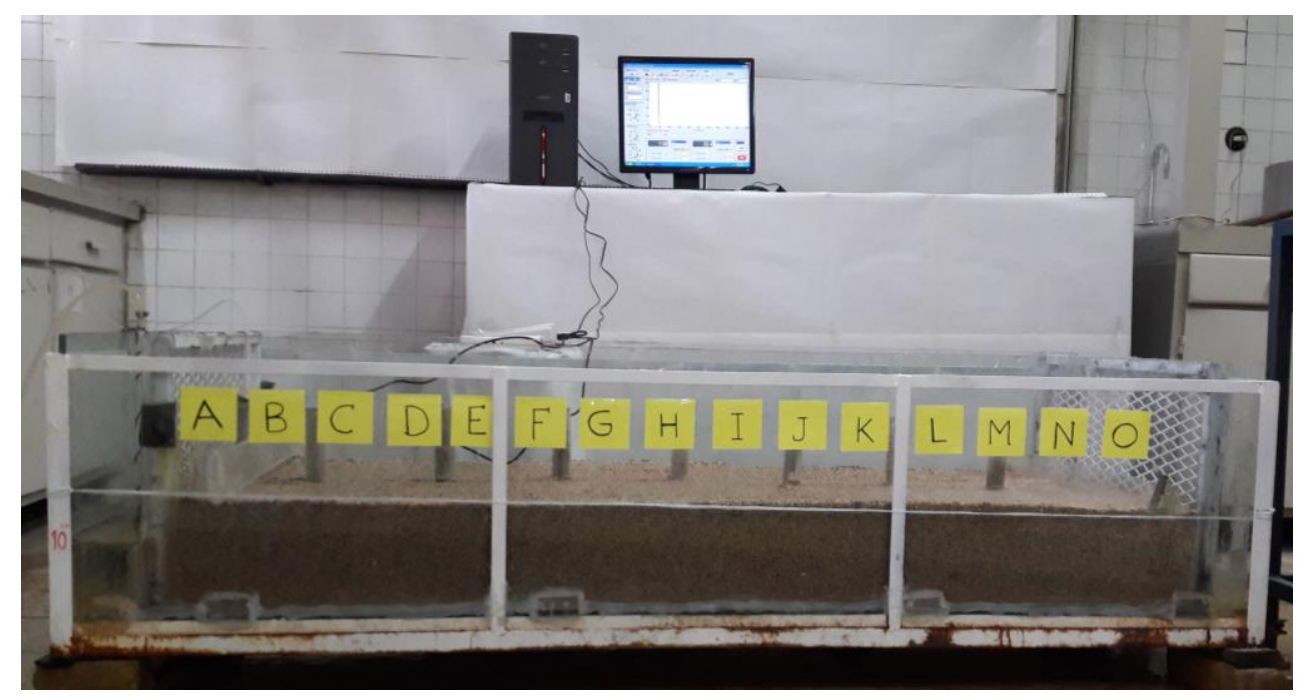

Figure 1: Laboratory Model

In the present study, salt $(\mathrm{NaCl})$ was used as a tracer. This tracer is easily soluble in water, cheap, available, environmentally friendly, and it is also possible to monitor its expansion in the model easily by an EC meter device. According to the results of the initial tests, which were carried out in this research study, the selected concentrations of tracer were equal to $5,7.5$ and $10 \mathrm{~g} / \mathrm{l}$. The results of $5 \mathrm{~g} / \mathrm{l}$ concentration tests are reported in this paper. To make a solution with $5 \mathrm{~g} / \mathrm{l}$ concentration, $12.71 \mathrm{grams}$ of $\mathrm{NaCl}$ was dissolved in a liter of distilled water. The tracer device comprised a wide low-depth reservoir, connective a flexible tube with a nozzle fixed at the end of the tube. The above mentioned nozzle had a valve to achieve a constant of tracer discharge. The nozzle was fixed at the central point of the model top-wide, inside the constant level head-water and closed to the soil; to release the tracer freely penetrates into the soil with a discharge of $2 \mathrm{ml} / \mathrm{s}$. The sand was saturated with the constant head-water before releasing of the tracer. It worth noting that, all experiments were repeated three times to obtain reliable data. 


\subsection{Fick's law and its analytical solution}

In the context of the transmission of contamination in a saturated porous medium the Fickian second law is used. If the flow of groundwater is two-dimensional and moves in the direction of the $\mathrm{x}$ axis with a uniform velocity $\mathrm{U}$, then the Fickian law can be presented as follows:

$$
D_{x} \frac{\partial^{2} x}{\partial x^{2}}+D_{y} \frac{\partial^{2} y}{\partial y^{2}}-U \frac{\partial C}{\partial x}=\frac{\partial C}{\partial t}
$$

Where;

$$
D_{x}=\alpha_{l} * U
$$

$$
D_{y}=\alpha_{t} * U
$$

and Dx is the longitudinal dispersion coefficient, Dy is the transverse dispersion coefficient, $\alpha \mathrm{l}$ is the longitudinal dispersion, $\alpha$ is the transverse dispersion, $U$ is the velocity in the direction of the axis $\mathrm{x}$ and $\mathrm{C}$ is the concentration.

In this research study, an analytical solution method was used to obtain scattering coefficients. The relevant equation of the analytic solution for the case of a source of pointwise and continuous pollutant is as follows:

$$
\mathrm{C}(\mathrm{x}, \mathrm{y}, \mathrm{t})=\mathrm{XY}
$$

where:

$$
\begin{aligned}
& X(\mathrm{x}, \mathrm{t})=\frac{\mathrm{C}_{0}}{2} \operatorname{erf}\left(\frac{x-U t}{2 \sqrt{D_{x} t}}\right) \\
& Y(y, t)=0.5\left\{\operatorname{erf}\left[\frac{y+0.5 B}{2 \sqrt{D_{y} x / U}}\right]-\operatorname{erf}\left[\frac{y-0.5 B}{2 \sqrt{D_{y} x / U}}\right]\right\}
\end{aligned}
$$

$\mathrm{B}$ is the width of the source of the pollutant, and other parameters have the same concepts as before.

\section{Results and Discussion}

The collected and recorded EC data were converted to concentration, using the following formula.

$$
p p m=a(E C)^{b}
$$

To obtain the values of $a$ and $b$, solutions were first prepared with different concentrations, in the laboratory, and the relevant EC values were then recorded for each solution. Then, the coefficients were calculated by plotting the graph and the fitting of the exponential function curve as presented below. 
Estimation of Longitudinal and Transverse Dispersion Coefficients in Saturated ... K. Ebrahimi et al.

$$
p p m=138.77(E C)^{1.1544}
$$

After converting data and calculating the concentration values at different times, considering that the data were recorded by the device with fluctuations and jumps, first all the concentration data were taken from the moving average with a period of 7 seconds and then using MATLAB software concentration graphs were plotted, involving Fourier equations or 9th order equations, see figure two below.

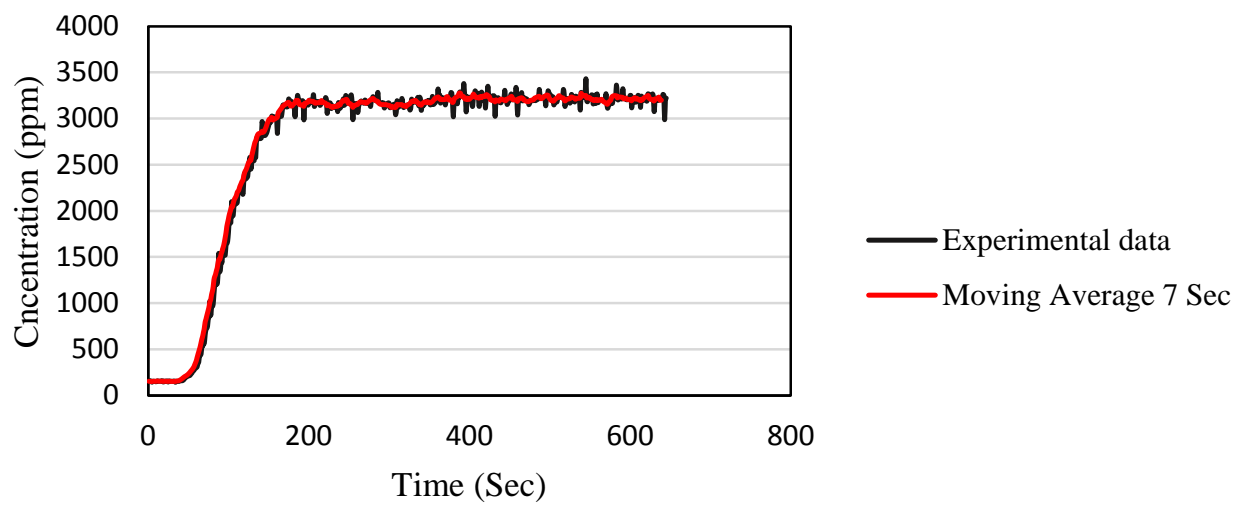

Figure 2: Concentration against time in section B (see Figure 1)

According to figure 2, the contaminant has been reached to the first recording point (well which installed at section B, Fig. 1) after approximately 50 seconds and then the concentration has increased dramatically. Following by that and after about 200 seconds, the contamination increased to a constant level of 3500ppm. The result of the analytic solution using Fick's law (Equ. 4) showed that has a Fourier series relationship type. It was used to obtain the analytical data and has a shape similar to the figure 2, i.e. s-curve. Also, as previously mentioned, the measured data were compared with the Fourier series using Matlab software and the comparison gave an encouraging level of agreement.

The following equation was then used, Zoe and Ekstine (1995) which is based on a statistical study of laboratory data.

$$
\alpha_{L}=0.83(\log L)^{2.414}
$$

where $\alpha_{l}$ and $\mathrm{L}$ both have similar unites of $m$.

These data were compared with the actual values obtained from the tests, and the relevant value of RMSE was calculated. Using the solver tool, by changing the longitudinal and transverse fragmentation values, the lowest RMSE and optimal scatter coefficients were calculated.

The results of the inverse solution for longitudinal and transverse dispersion coefficients are presented in table 1 , below.

\begin{tabular}{ccc}
\hline Concentration of Tracer $(\mathrm{g} / \mathrm{l})$ & $\mathrm{D}_{\mathrm{x}}\left(\mathrm{m}^{2} / \mathrm{s}\right)$ & $\mathrm{D}_{\mathrm{y}}\left(\mathrm{m}^{2} / \mathrm{s}\right)$ \\
\hline 5 & $3.362 * 10^{-6}$ & $6.583^{-6} 10^{-7}$ \\
\hline
\end{tabular}

Table 1: Distribution Coefficients 
Estimation of Longitudinal and Transverse Dispersion Coefficients in Saturated ... K. Ebrahimi et al.

These results are similar to Olsen's and Gaussian results.

Also with applying the Surfer software, the diffused tracer was graphically plotted, which is illustrates in Figure 3.

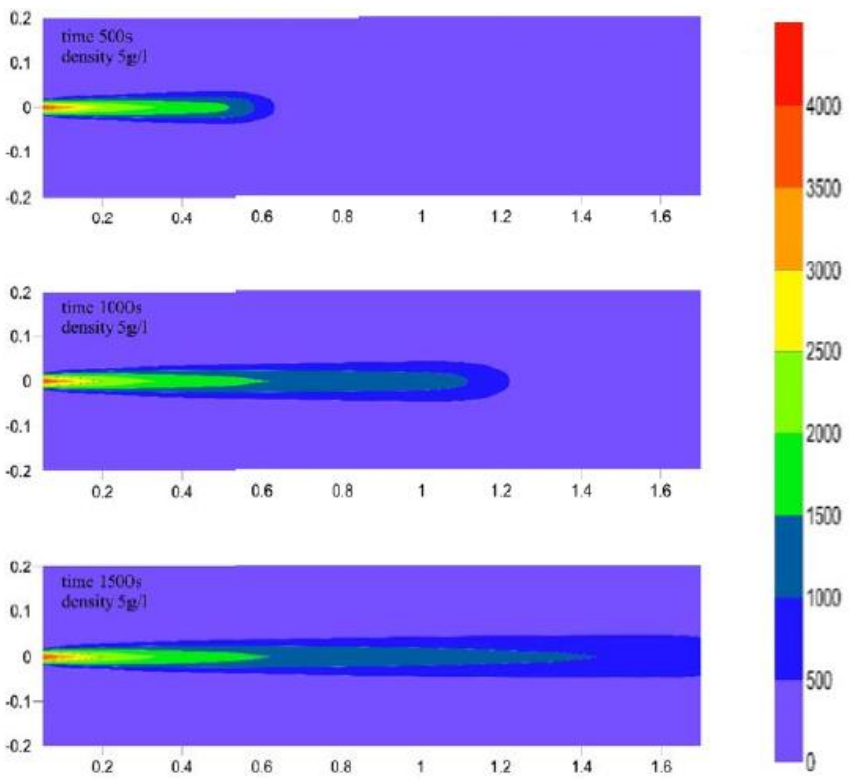

Figure 3: Tracer distribution at 500s, 100s, 1500s, concentration of $5 \mathrm{~g} / \mathrm{l}$

\section{Summary and Conclusions}

A laboratory model was constructed to study the movement of a tracer in saturated porous media, to estimate the longitudinal and transverse dispersion coefficients. Non-cohesive sands were used to make the porous media surroundings with a grain diameter of $1-2.5 \mathrm{~mm} . \mathrm{NaCl}$ with concentrations of $5,7.5$ and $10 \mathrm{~g} / \mathrm{l}$ were used as a tracer. The expansion of the salty plume was monitored using an ECmeter apparatus and the EC data were recorded. The collected and recorded EC data were used to calculate the tracer concentration data in different points over time, in gridded points. The measured data were compared with values which were obtained from the analytical solution based on Fickian second law. This comparison demonstrated the accuracy of the obtained results with an encouraging level of agreement. According to the results of the $5 \mathrm{~g} / \mathrm{l}$ of the tracer, the obtained values of the longitudinal and transverse coefficients are equal to $3.36 \mathrm{e}-6$ and $6.58 \mathrm{e}-7 \mathrm{~m} 2 / \mathrm{s}$, respectively.

\section{Acknowledgements}

The authors would like to acknowledge University of Tehran for providing the necessary facilities for conducting this research and preparing the related articles. 
Estimation of Longitudinal and Transverse Dispersion Coefficients in Saturated ... K. Ebrahimi et al.

\section{References}

Ye, M.S.Y., Experimental Investigation of Plume Dilution in Three-Dimensional Porous Media, Eberhard Karls Universität Tübingen 2016.

Marouf Pour E, Moazed H, Kashkouli H, Moahamad Vali Samani H. Laboratory Investigation of The Effect of the Sampling Method in Tracer Experiments on The Dispersion Coefficient of The Aquifer, JWSS;12 (46) :435-446, 2009.

Ayotamuno, M. J., Contaminant Transport and Immobilisation in Stratified Sands, PhD Thesis, University of Birmingham, UK, 1999.

Torkamani, S and Shayegan, J. "Modeling Dissolved Contamination Dispersion in Groundwater Resulting from a Contaminant Source Having non-Constant Leakage", 11th National Iranian Chemical Engineering Cngress, 2006.

Harbaugh, A.W., MODFLOW-2005, the US Geological Survey modular ground-water model: the ground-water flow process, US Department of the Interior, US Geological Survey Reston, VA, USA, 2005.

Khaledi, H and Tabatabaei, H. "Introduction to model and its applications in Irrigation and Drainage, "Modeling Training Workshop on Irrigation and Drainage, 2005.

Eftekhari, H. R. "Simulation of groundwater flow and distribution and contaminant transport in the plains Seyedan and Farooq", Master Thesis, Department of Civil Engineering, University of Shiraz, 2011.

Gaganis, P., Evaluation of dispersion coefficients from visualization experiments in artificial porous media. Journal of Hydrology, 307(1): p. 79-91, 2005.

McNeil, J, G. Oldenborger, and R. Schincariol, Quantitative imaging of contaminant distributions in heterogeneous porous media laboratory experiments. Journal of contaminant hydrology, 84(1): p. 36-54, 2006.

Olsson, $\AA$ and P. Grathwohl, Transverse dispersion of non-reactive tracers in porous media: A new nonlinear relationship to predict dispersion coefficients, Journal of contaminant hydrology, 92(3): p. 149-161, 2007.

Rolle, M., et al., Experimental investigation and pore-scale modeling interpretation of compoundspecific transverse dispersion in porous media, Transport in porous media, 93(3): p. 347-362, 2012.

Citarella, D., et al, Evaluation of dispersivity coefficients by means of a laboratory image analysis, Journal of contaminant hydrology, 2015. 172: p. 10-23, 2015.

Priya, M., et al, Study on Two Dimensional Dispersion of Pollutants through Porous Media, International Journal of Innovative Research in Engineering \& Management (IJIREM), 3(1), 2015.

Fadaei Tehrani, M.R., R. Feizy, and H. Jahanian, New Approach for Approximation of Dispersivity in Porous Media, Journal of Structural Engineering and Geo-Techniques, 6(2), 2016.

Ebrahimi, K., Development of an integrated free surface and groundwater flow model, PhD thesis, Cardiff University, UK, 2004.

Nazem, M. R; Ebrahimi, K; Liaghat, A; Araghinejad, S. A laboratory study of Tracer movement in saturated stat, involving physical model. $4^{\text {th }}$ International Conference on Environmental Planning and Management. Tehran, IRAN, 2017.

Danelson, R. E, and P. L, Sutherl, Methods of Soil Analysis, Part 1, Physical and Mineralogical Methods, Madison WI, Pp (1986): 635-662, 1986.

Fetter, C.W. and C. Fetter, Contaminant hydrogeology, Prentice hall New Jersey, Vol. 500. 1999.

Aggelopoulos, C. and C. Tsakiroglou, Study of the longitudinal dispersion coefficient of soils as related to the variability of local permeability, water, air, and soil pollution, 185(1-4): p. 223-237, 2007.

Haynes, W. M. (Ed.), CRC handbook of chemistry and physics. CRC press, 2014. 
Estimation of Longitudinal and Transverse Dispersion Coefficients in Saturated ... K. Ebrahimi et al.

Bureau of Engineering and Technical Criteria for Water and Wastewater, Ministry of Energy, "Instruction For Application of Groundwater Tracing Methods in Alluvial Aquifers Studies No.522", Tehran, IRAN, (2011).

Mahmoodian-Shooshtari, M. "Hydraulics of Groundwater", Shahid Chamran University of Ahvaz, Ahvaz, 2013.

$\mathrm{Xu}, \mathrm{M}$. and Y. Eckstein, Use of weighted least squares method in evaluation of the relationship between dispersivity and field scale. Ground Water, 33(6): p. 905-908, 1995.

Cirpka, O. A., Determination of transverse dispersion coefficients from reactive plume lengths. Ground Water, 44(2): p. 212-221, 2006. 